\title{
Editorial: Multimodal Mating Signals: Evolution, Genetics and Physiological Background
}

\author{
Astrid T. Groot ${ }^{1 *}$, Varvara Vedenina ${ }^{2}$ and Emily Burdfield-Steel ${ }^{1}$ \\ ${ }^{1}$ Institute for Biodiversity and Ecosystem Dynamics, University of Amsterdam, Amsterdam, Netherlands, ${ }^{2}$ Institute for \\ Information Transmission Problems, Russian Academy of Sciences (RAS), Moscow, Russia
}

Keywords: natural selection, sexual selection, visual signals, acoustic signals, chemical signals, multimodal integration, learning, environmental interactions

Editorial on the Research Topic

Multimodal Mating Signals: Evolution, Genetics and Physiological Background

When communicating vital information, such their attractiveness to potential mating partners or their unpalatability to predators, species are not restricted to signaling in a single mode. Instead they may produce signals in two or more modes, aka multimodal signaling. Multi-modal (pre)mating signals may be a combination of acoustic and visual signals, such as those used by birds (Ota et al., 2015; Cooney et al., 2018), frogs (Halfwerk et al.), and fish (de Jong et al.). In addition, combinations of acoustic and chemical signals are used by myrmecophilus butterflies in their complex parasitic interactions with ants (Casacci et al.), and many aposematic species use a combination of visual and

OPEN ACCESS

Edited and reviewed by:

Elise Huchard,

UMR5554 Institut des Sciences de l'Evolution de Montpellier (ISEM), France

*Correspondence: Astrid T. Groot a.t.groot@uva.nl

Specialty section:

This article was submitted to Behavioral and Evolutionary Ecology,

a section of the journal

Frontiers in Ecology and Evolution

Received: 18 November 2020

Accepted: 09 December 2020

Published: 12 January 2021

Citation:

Groot AT, Vedenina $V$ and Burdfield-Steel E (2021) Editorial: Multimodal Mating Signals: Evolution,

Genetics and Physiological

Background.

Front. Ecol. Evol. 8:630957.

doi: 10.3389/fevo.2020.630957 chemical to ward off predators (Rojas et al.). Thus, multiple signals may interact with each other, and understanding the evolutionary pressures on these signals requires a thorough understanding of these interactions.

In general, signals can be under sexual and natural selection pressures. Rojas et al. discuss in detail how the complex interplay between natural and sexual selection can influence aposematic displays. Species with high within-population variability are particularly excellent models to determine how different selection forces affect the evolution of warning signals, but unfortunately there are not many studies focusing on intraspecific variation within and between populations that consider both natural and sexual selection (Rojas et al.). One exception is poison frogs, where the most well-defended males are also the most attractive ones (Maan and Cummings, 2008), so that natural and sexual selection work in concert. In invertebrates, such as beetles, Heliconius butterflies and day-flying moths, studies focus mostly on predator-imposed frequency-dependent selection on color variation. How variation in color interacts with chemical signals, such as those used as chemical defenses against predators, and sex pheromones, is now starting to be investigated (Gordon et al., 2015; Rojas et al., 2019).

The level and extent of natural and sexual selection pressures depends on the receivers of the signal. When under sexual selection, signals can be received by both competitors (intra-sexual communication signals) and by the choosing sex (inter-sexual communication signals). Vedenina and Shestakov studied the interplay between female preferences and male-male interactions in the cricket Gryllus bimaculatus to evaluate which sensory modalities are an indicator of male dominance status. As females mounted winners and losers equally often, the authors bring forward the interesting, and generally ignored, point that signals used by females may differ from signals used by males. Similarly, Rajaraman et al. show that both males and females in the bushcricket Onomarchus uninotatus may signal to each other, albeit with different types of signals and different forms of responses: males have acoustic calls and respond to vibrating females with vibrotaxis, while 
females use vibratory signals and respond to males with phonotaxis or tremulation, depending on the distance between the sexes.

Compared to unimodal signals, multimodal signals are special in the sense that they may improve associative learning. As there is more information per unit time, interactions may be more efficient than each signal by itself (Rojas et al.). Multimodal signals may also be backup signals: if one channel is blocked, a receiver can still receive the message through the other channel (Halfwerk et al.). A nice example of a redundant signal is given by Deodhar and Isvaran in the lizard Psammophilus dorsalis, where males use behavioral as well as color signals, which are strongly correlated. However, as different signal traits were affecting competitors, mates, and predators differentially, different signal components may be less redundant, but rather influenced by multiple selection pressures.

Multimodal signals may also be multiple messages which can convey different information at the same time, for example species identity as well as intention to mate (Halfwerk et al.). Importantly, multimodal signal evolution depends not only on who the receivers are, but also how signals are processed by the receiver's sensory systems. Halfwerk et al. review the current state of knowledge on how multimodal signals are integrated, ranging from humans and other vertebrates to insects. Multimodal integration is well-studied in humans, but also prevalent in birds, bees, fish, insects and frogs. Since interactive perception may give different results than each signal separately, Halfwerk et al. plea for an integrated approach to assess multimodal percepts.

Complicating factors that may affect the evolution of multimodal communication include learning, as well as interactions with the biotic and abiotic environment. Dion et al. extensively reviewed evidence that learning affects the evolution of (pre)mating signals in spiders and insects, meaning that sexual interactions are modified after experience. This effect is also nicely shown by Bunting and Hedrick, who found that previous encounters between males in the cricket Gryllus integer alters the songs of dominant (winning) males, but not subordinate (losing) males. As Dion et al. show, both short and long term memory of previous experiences impact lifetime mating behaviors in many insect and spider species, and both can learn multiple types of

\section{REFERENCES}

Cooney, C. R., MacGregor, H. E. A., Seddon, N., and Tobias, J. A. (2018). Multimodal signal evolution in birds: re-examining a standard proxy for sexual selection. Proc. R. Soc. B Biol. Sci. 285:20181557. doi: 10.1098/rspb.2018.1557

Gordon, S. P., Kokko, H., Rojas, B., Nokelainen, O., and Mappes, J. (2015). Colour polymorphism torn apart by opposing positive frequencydependent selection, yet maintained in space. J. Anim. Ecol. 84, 1555-1564. doi: $10.1111 / 1365-2656.12416$

Maan, M. E., and Cummings, M. E. (2008). Female preferences for aposematic signal components in a polymorphic poison frog. Evolut. Int. J. Organ. Evol. 62, 2334-2345. doi: 10.1111/j.1558-5646.2008.00454.x

Ota, N., Gahr, M., and Soma, M. (2015). Tap dancing birds: the multimodal mutual courtship display of males and females in a socially monogamous songbird. Scient. Rep. 5:16614. doi: 10.1038/srep16614 information. However, almost all studies have focused on visual or acoustic or olfactory signals, instead of possible combinations of interactions, so that it remains unclear whether and how learning of multimodal signals could be involved in the evolution of multimodal signaling.

Finally, biotic and abiotic environmental factors may also influence multimodal signal evolution. de Jong et al. determined the effect of anthropogenically induced noise on courtship behavior in the painted goby (Pomatoschistus pictus), in which visual and acoustic signals are used by males to attract females. As female gobies paid more attention to visual than acoustic signals in noisy environments, this study brings forth the important point that selection pressures on multimodal signals may differ depending on the level and extent of interfering habitat backgrounds. Background interference can take many forms, from anthropogenically produced noise to naturally-occurring heterospecific signals. The fact that social environment affects multimodal signals is best illustrated by the complex multimodal signaling in myrmecophilous butterflies, as reviewed by Casacci et al. These butterflies have developed amazing obligate-parasitic life history strategies with Myrmica ants to fulfill their life cycle, and use visual, chemical and acoustic signals to attract and deceive ant workers. Casacci et al. put forward the appealing hypothesis that the amazing signal complexity between these butterflies and ants may have boosted the evolution of specialized life cycles, thereby increasing butterfly diversity.

In conclusion, the contributions presented in this Research Topic highlight the wide variety of possible mechanisms underlying multimodal signaling and mate preferences for multiple traits, and give several ideas for further research in this framework. To fully understand the evolution of multimodal signaling it is essential to investigate the genetic basis of the different signals and how these different signals are processed by the receiver's sensory systems, as well as identifying the interacting selection pressures acting on multimodal signals.

\section{AUTHOR CONTRIBUTIONS}

All authors listed have made a substantial, direct and intellectual contribution to the work, and approved it for publication.

Rojas, B., Mappes, J., and Burdfield-Steel, E. (2019). Multiple modalities in insect warning displays have additive effects against wild avian predators. Behav. Ecol. Sociobiol. 73:37. doi: 10.1007/s00265-019-2643-6

Conflict of Interest: The authors declare that the research was conducted in the absence of any commercial or financial relationships that could be construed as a potential conflict of interest.

Copyright (C) 2021 Groot, Vedenina and Burdfield-Steel. This is an open-access article distributed under the terms of the Creative Commons Attribution License (CC BY). The use, distribution or reproduction in other forums is permitted, provided the original author(s) and the copyright owner(s) are credited and that the original publication in this journal is cited, in accordance with accepted academic practice. No use, distribution or reproduction is permitted which does not comply with these terms. 